ANNALES

POLONICI MATHEMATICI

$\mathrm{XXIX}$ (1974)

\title{
Sur un Théorème de Schwerdtfeger
}

\author{
par J. Dieudonné (Nice)
}

Résumé. H. Schwerdtfeger a prouvé le théorème suivant: soit $\mathfrak{X}(t)$ une matrice carrée d'ordre $n$ dont les éléments sont fonctions analytiques d'une variable réelle $t$ dans un intervalle $I$. On suppose que pour tout $t \epsilon I$ les valeurs propres de $\mathfrak{X}(t)$ sont distinctes et que $\mathfrak{X}(t) \mathfrak{X}^{\prime}(t)=\mathfrak{X}^{\prime}(t) \mathfrak{X}(t)$ pour tout $t$; alors pour deux valeurs quelconques $t_{1}, t_{2}$ dans $I, \mathfrak{X}\left(t_{1}\right)$ et $\mathfrak{X}\left(t_{2}\right)$ sont permutables. Il est prouvé que co théorème est encore valable lorsqu'on suppose seulement que les éléments de $\mathfrak{X}(t)$ sont continûment différentiables.

1. Soit $t \rightarrow \mathfrak{X}(t)$ une application d'un intervalle $I$ de $\boldsymbol{R}$ dans l'espace des matrices carrées complexes d'ordre n. Supposons cette application dérivable, et telle que l'on ait

$$
\mathfrak{X}(t) \mathfrak{X}^{\prime}(t)=\mathfrak{X}^{\prime}(t) \mathfrak{X}(t) \quad \text { pour tout } t \in I \text {. }
$$

H. Schwerdtfeger a prouvé $\left({ }^{1}\right)$ que si, pour $t \in I, \mathfrak{X}(t)$ a toutes ses valeurs propres distinctes, il existe une matrice inversible constante $\mathfrak{P}_{0}$ telle que $\mathfrak{X}(t)=\mathfrak{P}_{0} \mathfrak{D}(t) \mathfrak{P}_{0}^{-1}$, où $\mathfrak{D}(t)$ est une matrice diagonale; cela entraîne en particulier que $\mathfrak{X}\left(t_{1}\right)$ et $\mathfrak{X}\left(t_{2}\right)$ sont permutables pour deux valeurs quelconques $t_{1}, t_{2}$ de $t \in I$; il a aussi donné des exemples montrant que la conclusion est en défaut si les valeurs propres de $\mathfrak{X}(t)$ ne sont pas toutes distinctes. Toutefois, sa démonstration suppose la fonction $t \rightarrow \mathfrak{X}(t)$ analytique dans $I$; je me propose de faire voir, par une autre méthode, que cette restriction est superflue, et qu'il suffit de supposer $\mathfrak{X}$ continûment différentiable.

2. Soient $\lambda_{1}\left(t_{1}\right), \lambda_{2}(t), \ldots, \lambda_{n}(t)$ les valeurs propres de $\mathfrak{X}(t)$. Comme elles sont toujours distinctes par hypothèse pour $t \epsilon I$ et que $I$ est simplement connexe, le théorème des fonctions implicites montre qu'il y a effectivement $n$ fonctions continûment différentiables $t \rightarrow \lambda_{k}(t)(1 \leqslant k \leqslant n)$ dans $I$ dont les valeurs, pour chaque $t$, sont les valeurs propres de $\mathfrak{X}(t)$ : il suffit de remarquer que si $z^{n}+f_{1}(t) z^{n-1}+\ldots+f_{n}(t)=0$ est l'équation caractéristique de $\mathfrak{X}(t)$, les points $(t, z) \in I \times C$ vérifiant cette relation forment

(1) H. Schwerdtfeger, Sur les matrices permutables avec leur dérivée, Univ. e Politecnico Turin, Rend. Sem. Mat. 11 (1952), p. 329. 
un revêtement de $I$. Posons $\mathfrak{D}(t)=\operatorname{diag}\left(\lambda_{1}(t), \ldots, \lambda_{n}(t)\right)$; les formules de Cramer montrent que pour tout $t_{0} \epsilon I$, il y a un voisinage $V$ de $t_{0}$ et une matrice inversible $\mathfrak{P}(t)$, fonction continûment dérivable dans $V$, tels que dans $V$, on ait

$$
\mathfrak{X}(t)=\mathfrak{P}(t) \mathfrak{D}(t) \mathfrak{P}(t)^{-1} .
$$

3. Ecrivons alors la relation (1) en substituant à $\mathfrak{X}$ sa valeur tirée de (2). Comme $\mathfrak{X}^{\prime}=\mathfrak{P}^{\prime} \mathfrak{D P}^{-1}+\mathfrak{B D}^{\prime} \mathfrak{P}^{-1}-\mathfrak{B D} \mathfrak{B}^{-1} \mathfrak{B}^{\prime} \mathfrak{P}^{-1}$, on obtient, après multiplication à droite par $\mathfrak{P}$ et à gauche par $\mathfrak{P}^{-1}$, et en remarquant que $\mathfrak{D}^{\prime} \mathfrak{D}=\mathfrak{D D}^{\prime}$,

$$
\mathfrak{P}^{-1} \mathfrak{P}^{\prime} \mathfrak{D}^{2}+\mathfrak{D}^{2} \mathfrak{P}^{-1} \mathfrak{P}^{\prime}=2 \mathfrak{D} \mathfrak{P}^{-1} \mathfrak{P}^{\prime} \mathfrak{D}
$$

ou, en posant $\mathfrak{U}=\mathfrak{P}^{-1} \mathfrak{P}^{\prime}$,

$$
(\mathfrak{U} \mathfrak{D}-\mathfrak{D} \mathfrak{U}) \mathfrak{D}=\mathfrak{D}(\mathfrak{U} \mathfrak{D}-\mathfrak{D} \mathfrak{U}) .
$$

Mais une matrice ne peut permuter avec $\mathfrak{D}$ que si elle est elle-même diagonale; et si l'on écrit que $\mathfrak{U D}-\mathfrak{D} \mathfrak{U}$ est diagonale, on trouve aussitôt que $\mathfrak{U}$ elle-même doit être diagonale, en vertu $d u$ fait que les $\lambda_{k}(t)$ sont toutes distinctes (l'algèbre de Lie des matrices diagonales est sa propre normalisatrice dans l'algèbre de Lie des matrices d'ordre $n$ ). On en conclut que dans $V$, il y a $n$ fonctions continûment dérivables $g_{k}(t)$ telles que, si l'on pose $\mathfrak{B}(t)=\left(p_{j k}(t)\right)$, on ait

$$
p_{j k}^{\prime}(t)=p_{j k}(t) g_{k}(t) \quad \text { pour } 1 \leqslant j, k \leqslant n
$$

et par suite, en posant $G_{k}(t)=\exp \left(\int g_{k}(t) d t\right)$,

$$
p_{j k}(t)=c_{j k} G_{k}(t)
$$

où $c_{j k}$ est une constante, ce qui s'écrit aussi $\mathfrak{P}(t)=\mathfrak{C} \mathfrak{G}(t)$, où $\mathfrak{C}=\left(c_{j k}\right)$ et $\mathfrak{G}(t)=\operatorname{diag}\left(G_{1}(t), \ldots, G_{n}(t)\right)$; mais alors on a $\mathfrak{X}(t)=\mathbb{C D}(t) \mathbb{C}^{-1}(\mathbb{C}$ étant nécessairement inversible puisque $\mathfrak{P}(t)$ l'est).

Il reste à remarquer que si $V_{1}, V_{2}$ sont deux intervalles ouverts de $I$ d'intersection non vide, et si $\mathfrak{X}=\mathfrak{P}_{1} \mathfrak{D P}_{1}^{-1}$ dans $V_{1}$, et $\mathfrak{X}=\mathfrak{P}_{2} \mathfrak{D P}_{2}^{-1}$ dans $V_{2}$, où $\mathfrak{P}_{1}$ et $\mathfrak{P}_{2}$ sont des matrices constantes, on a aussi $\mathfrak{X}=\mathfrak{P}_{1} \mathfrak{D P}_{1}^{-1}$ dans $V_{2}$ : en effet, si $t_{0} \in V_{1} \cap V_{2}, \mathfrak{P}_{2}^{-1} \mathfrak{P}_{1}$ doit permuter avec $\mathfrak{D}\left(t_{0}\right)$, done est une matrice diagonale $\mathfrak{L}$; mais comme $\mathfrak{L}$ et $\mathfrak{D}(t)$ permutent pour tout $t$, cela établit notre assertion. On conclut en recouvrant $I$ par des voisinages où une expression (2) de $\mathfrak{X}$ est valable.

Reçu par la Rédaction le 15. 3. 1973 